\title{
Aplicación móvil para niños con dificultades de aprendizaje en la automatización del proceso de reconocimiento de palabras
}

\author{
Carlos Máñez-Carvajal* y Jose Francisco Cervera-Mérida \\ Universidad Católica de Valencia San Vicente Mártir, c/ Sagrado Corazón, 5. 46110 Godella-España \\ (correo-e: carlos.manez@ucv.es; josefran.cervera@ucv.es) \\ * Autor a quien debe ser dirigida la correspondencia.
}

Recibido Mar. 1, 2021; Aceptado Abr. 24, 2021; Versión final Jun. 13, 2021, Publicado Oct. 2021

\begin{abstract}
Resumen
El presente trabajo tiene como objetivo el desarrollo de una aplicación móvil para facilitar el aprendizaje de la lectura y los procesos de reconocimiento automático de palabras y acceso al léxico a niños con dificultades de aprendizaje. Para llevar a cabo el desarrollo se utilizó Basic4android, un software adecuado para crear aplicaciones compatibles en una tableta con sistema operativo Android. La aplicación contiene un vocabulario de 1500 palabras y tiene el aspecto de juego. Está basada en los principios de activación de la ruta directa de reconocimiento de palabras o ruta global y en la anticipación cognitiva. El resultado de este desarrollo informático permite que los docentes puedan controlar con facilidad las variables determinantes en el procesamiento cognitivo de los ejercicios. Se concluye que la aplicación móvil desarrollada aquí puede ayudar en la automatización del proceso de reconocimiento de palabras a niños con dificultades de aprendizaje de lectura.
\end{abstract}

Palabras clave: tecnología educativa; aplicación móvil; software educativo; dificultades de aprendizaje; dislexia

\section{Mobile application for children with learning difficulties in automating the word recognition process}

\begin{abstract}
The aim of this study is to develop a mobile application that facilitates learning to read and that facilitates the processes of automatic word recognition and lexical access for children with learning difficulties. The Basic4android software, suitable for creating compatible applications on a tablet with Android operating system, was used to develop the application. The application contains a vocabulary of 1500 words and the appearance of a game. It is based on the principles of direct path activation of word recognition or global path and cognitive anticipation. The mobile application allows teachers to easily control variables in the cognitive processing of exercises. It is concluded that the mobile application developed here can help in automating the process of word recognition for children with learning difficulties in reading.
\end{abstract}




\section{INTRODUCCIÓN}

Las Tecnologías de la Información y la Comunicación (TIC) ponen a nuestra disposición una amplia gama de dispositivos hardware como computadoras, tabletas y teléfonos inteligentes que permiten realizar nuestra vida cotidiana de una manera más sencilla. En la última década, el desarrollo o uso de aplicaciones informáticas ha aumentado de manera exponencial independientemente del sector, industria o tipo de dispositivo empleado (Castro-Rivera et al., 2020; Máñez-Carvajal, 2020; Orellana et al., 2020). El incremento del número de usuarios de dispositivos móviles y los avances en tecnología no han pasado desapercibidos en el contexto educativo, el aprendizaje móvil ofrece nuevas estrategias, procedimientos y procesos formativos a niños y adultos. Estas tecnologías son fáciles de transportar, resultan relativamente baratas y favorecen el aprendizaje eliminando las fronteras entre la educación formal y la no formal, las restricciones temporales y de ubicación. Cada vez son más numerosos los proyectos que demuestran que las tecnologías móviles constituyen un medio excelente para ampliar las oportunidades educativas de alumnos que tal vez no tengan acceso a una enseñanza de alta calidad (West y Vosloo, 2013).

El uso de las tabletas supone un cambio en la manera de aprender de los estudiantes, puede aumentar su deseo de participación e incrementar su motivación e interés. El desarrollo y uso de aplicaciones posibilita crear soluciones de enseñanza flexibles, un acceso a la información y un aprendizaje personalizado a las necesidades de los alumnos en diferentes situaciones y dispositivos. Además, la aparición de entornos de desarrollo como Eclipse, Basic4android o Android Studio (entre otros) que permiten crear aplicaciones para este tipo de dispositivos con menor coste y mayor facilidad de desarrollo ha contribuido a este sustancial auge en el uso de estas tecnologías. Como consecuencia de ello, los estudios y desarrollos informáticos realizados por docentes e investigadores son muy numerosos. Aquellos que se encuentran focalizados en el ámbito educativo, se han centrado principalmente en analizar la mejora del aprendizaje y la percepción de estudiantes y profesores sobre el uso de estas tecnologías independientemente de la materia tratada (Fuentes et al., 2019; Gou y Dezuanni, 2018; Morales et al., 2020).

En los últimos años se han desarrollado aplicaciones móviles específicamente diseñadas para personas con dificultades específicas de aprendizaje de la lectura o dislexia. Este trastorno afecta entre un $2 \%$ y un $5 \%$ de la población mundial, se manifiesta como una dificultad severa para el aprendizaje y no se debe a deficiencia intelectual, déficits visuales o auditivos no corregidos, trastornos mentales o neurológicos, adversidad psicosocial, falta de dominio del lenguaje o de instrucción académica adecuada. Se diagnostica con los criterios del DSMV (Asociación Estadounidense de Psiquiatría, 2014) durante los primeros cursos de la escolarización, provoca fracaso escolar y persiste toda la vida, aunque algunas personas pueden compensarlo y alcanzar el éxito académico. Las personas con dislexia tienen dificultades para la automatización de la lectura y escritura, con manifestaciones diferentes a lo largo del ciclo vital (Galuschka et al., 2020). La mayoría son muy lentos para identificar las palabras y tienen severos problemas para aprender la ortografía. En función de su severidad puede influir decisivamente en el resto de los aprendizajes escolares y tener consecuencias psicológicas y sociales. Algunas de las aplicaciones informáticas desarrolladas solamente realizan pruebas para su detección y otras abordan estrategias de mejora del aprendizaje en los dominios del lenguaje escrito y del cálculo matemático o de enriquecimiento en factores que se consideran causales, como la memoria de trabajo, la conciencia fonológica o las habilidades de automatización y nombramiento rápido.

La teoría actual sobre como los seres humanos reconocen los caracteres escritos y los asocian a palabras que tienen almacenadas en su memoria se denomina teoría de la doble ruta (Snowling y Hulme, 2005). La ruta fonológica, también Ilamada ruta indirecta, permite identificar cada letra y asociarla a un sonido. La palabra se reconoce combinando uno a uno sus integrantes de izquierda a derecha, o en el orden que corresponda según la cultura, y descodificando los signos gráficos a sus correspondientes sonidos para acceder a su pronunciación y significado. La ruta léxica o ruta directa, permite a la persona reconocer de forma global toda la palabra escrita y asociarla a su significado y también a cómo se pronuncia. La ruta indirecta resulta imprescindible para leer palabras que nunca han sido vistas o palabras muy poco frecuentes. La ruta directa, por el contrario, es muy eficiente con las palabras habituales. Cuando aprenden a leer los niños suelen usar preferentemente la ruta indirecta y, en la medida que se convierten en expertos, la ruta directa les aporta rapidez y facilidad para el reconocimiento de palabras. Los buenos lectores reconocen de forma global y automática gran número de palabras. Un lector típico necesita muy pocas exposiciones a una palabra nueva (Martínez-García et al., 2019), que tendrá que leer mediante la ruta indirecta un número suficiente de veces para que pueda ser reconocida automáticamente mediante la ruta directa.

Los niños con dificultades específicas de aprendizaje suelen tener serios problemas para el reconocimiento de las palabras, tanto en la identificación de los componentes de las palabras como en su reconocimiento global. Según su tipología, estas dificultades pueden ser mayores para el uso efectivo de la ruta directa, de la indirecta o de ambas. Por ese motivo, los educadores y terapeutas que trabajan ellos aplican estrategias para mejorar el reconocimiento de palabras por ambas rutas de lectura (Peters et al., 2019). 
Skiada et al. (2014) implementaron una aplicación compatible con dispositivos Android para fomentar el aprendizaje y ayudar a los niños mejorar algunas de sus habilidades fundamentales, como la comprensión de la lectura, la codificación ortográfica, la memoria a corto plazo y la resolución de problemas matemáticos. Tariq y Latif (2016) diseñaron una aplicación móvil para mejorar el rendimiento del aprendizaje en la escritura de niños con dislexia. El estudio mostró que los participantes se familiarizaron con las características de la aplicación en un corto período de tiempo y que utilizaron la aplicación sin esfuerzo. Además, el estudio reveló que los gráficos como los elementos textuales de la aplicación ayudaron a los estudiantes con dislexia a concentrarse, a evitar las distracciones y a dirigir su atención hacia la pantalla del dispositivo.

Las aplicaciones informáticas se han empleado con éxito tanto en la detección de la dislexia en niños (Alonzo et al., 2020), en el tratamiento educativo para remediarla (Chiang y Liu, 2011) o como asistentes que proporcionan ayuda en el desempeño académico diario de las personas que la padecen (Dawson et al., 2019). Su eficacia ha sido demostrada en aplicaciones informáticas diseñadas para mejorar el aprendizaje del acceso al léxico mediante estrategias que favorecen el reconocimiento de las sílabas en idiomas con ortografía regular, tal como son el italiano y el español (Tressoldi et al., 2007). También se ha probado la eficacia de aplicaciones que desarrollan la conciencia fonológica y la relación entre las letras y los sonidos (Kast et al., 2011) y también en otras que entrenan el reconocimiento del léxico mediante estrategias de activación de la ruta léxica y subléxica a la vez (Tucci et al., 2015).

En el proceso de elaboración de software existen actividades estructurales genéricas que son aplicables a todos los proyectos de desarrollo de software, sin importar su tamaño o complejidad (Alarcón-Aldana et al., 2016; Cáceres y Ossandón, 2018; Choque-Tolmo et al., 2020). Pressman (2010) las clasifica en: 1) Comunicación (antes de comenzar cualquier trabajo tiene importancia crítica comunicarse y colaborar con el cliente para definir las características y funciones del software); 2) Planeación (se define el trabajo de ingeniería de software y se describen las tareas técnicas por realizar, los riesgos probables, los recursos que se requieren y una programación de las actividades); 3) Modelado (se crea un modelo a fin de entender mejor los requerimientos del software y el diseño que los satisfará); 4) Construcción (esta actividad combina la generación de código y las pruebas que se requieren para descubrir errores en éste); y 5) Despliegue (el software como entidad completa o como un incremento parcialmente terminado, se entrega al consumidor que lo evalúa y da retroalimentación).

Estas actividades se integran de una u otra manera en los distintos modelos de diseño de software y sus variantes. El modelo en cascada es el más extendido, utiliza una metodología sistemática y una estructura lineal. Otros modelos emplean metodologías y estructuras diferentes, como el modelo evolutivo (Figura 1) que consiste en desarrollar rápidamente un sistema inicial a partir de especificaciones abstractas del cliente. Posteriormente este sistema se va refinando, basándose en las peticiones del cliente para elaborar un producto final que satisfaga sus necesidades (Sommerville, 2005). Una ventaja que presenta este modelo es que las actividades de especificación, desarrollo y validación se entrelazan con una rápida retroalimentación entre ellas. En la producción de sistemas pequeños, un enfoque evolutivo para el desarrollo de software es más efectivo pues satisface las necesidades más inmediatas de los clientes y permite desarrollar versiones intermedias cada vez más completas y complejas, hasta llegar a la versión final del programa. El objetivo principal de este trabajo es el desarrollo de una aplicación compatible en una tableta con sistema operativo Android destinada a niños con dificultades de aprendizaje de la lectura, que tiene como objetivo ayudar en el proceso de reconocimiento automático de palabras.

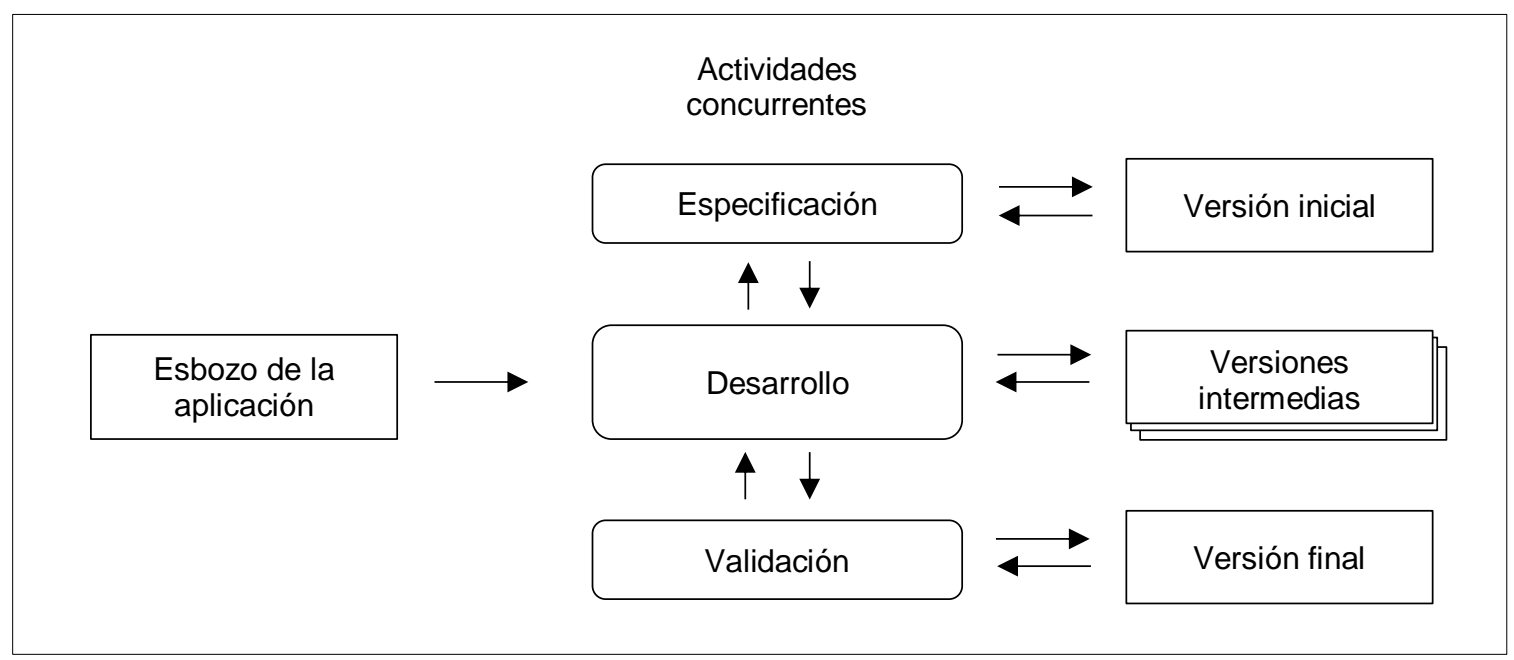

Fig. 1. Esquema del modelo evolutivo, adaptado de Sommerville (2005) 


\section{MATERIALES Y MÉTODOS}

Se detallan los materiales y métodos empleados para el desarrollo de la aplicación. Esto se presenta en cuatro subsecciones: análisis de requerimientos, herramienta empleada, vocabulario de la aplicación, y el procedimiento

\section{Análisis de requerimientos}

En la fase de comunicación se especificaron las funciones, características y requerimientos del software implementado: 1) La aplicación debe ser compatible en una tableta con un sistema operativo Android; 2) Se debe evitar la repetición de los ejercicios; 3) En la configuración del programa, el educador debe tener la opción de usar un anticipador de las palabras que presenta al usuario; 4) El desarrollo debe tener en cuenta la presentación de las palabras en milisegundos y 5) El programa debe mostrar el progreso del alumno.

\section{Herramienta empleada}

La aplicación fue desarrollada empleando Basic4android que permite crear aplicaciones compatibles con el sistema operativo Android. Esta herramienta emplea BASIC, un lenguaje de programación de alto nivel cuya filosofía de diseño enfatiza la facilidad de uso.

\section{Vocabulario de la aplicación}

Las palabras empleadas en el programa han sido seleccionadas, ordenadas y clasificadas en 12 conjuntos en virtud de su frecuencia de uso según el "Vocabulario Básico de EGB" Espasa Calpe-MEC. Cada conjunto tiene, aproximadamente, 125 palabras. Un conjunto de 382 adverbios no acabados en "mente" y dos conjuntos de palabras función (preposiciones, conjunciones, relativos, demostrativos y artículos). Las palabras más frecuentes se recopilaron en el conjunto 1 y las menos frecuentes en el 12.

\section{Procedimiento}

Para llevar a cabo el proyecto se realizó un estudio previo tomando como referencia las características y funciones del software especificadas por el equipo pedagógico. Se analizaron varias aplicaciones comerciales existentes para analizar el sistema de progresión del alumno, así como la presentación de refuerzos auditivos y visuales al acertar un ejercicio (Máñez-Carvajal y Máñez, 2020). Debido a las características del software, se optó por aplicar el modelo evolutivo de software realizando un esbozo inicial de la aplicación.

Para dar respuesta a las especificaciones de diseño se empleó la herramienta visual designer que simplifica el proceso de construcción de la interfaz de usuario en teléfonos y tabletas con diferentes tamaños de pantalla (Figura 2).

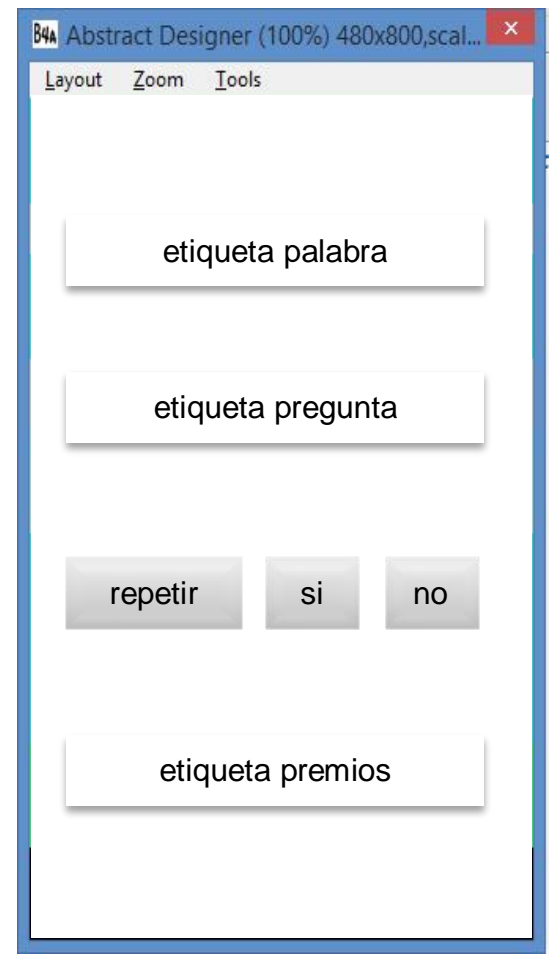

Fig. 2. Herramienta Visual designer 
Esta versión inicial del programa se presentó al equipo pedagógico y se refinaron aspectos pedagógicos y de diseño de la aplicación. En cuanto al desarrollo informático, los diferentes sonidos de acierto y error fueron almacenados en vectores de almacenamiento de datos. Se consideró hacer uso de bases de datos para almacenar el contenido de cada conjunto de palabras, sin embargo, teniendo en cuenta el tipo de desarrollo se optó por utilizar ficheros de texto. Ello permite al equipo pedagógico generar nuevos conjuntos de palabras con suma facilidad, aumentando o modificando las palabras de cada conjunto sin variar el código fuente del programa. Se diseñaron los procedimientos necesarios para evitar la repetición de las palabras en la presentación de los ejercicios. En este caso, se utilizaron funciones para generar números aleatorios y vectores auxiliares para almacenar los resultados y así poder determinar el acierto o error del alumno. Se utilizó un objeto de tipo caja de verificación para incluir la opción del anticipador y un objeto de entrada de texto para gestionar la presentación temporal de los ejercicios haciendo uso de objetos temporales. En ambos casos, los resultados se almacenaron en variables para guardar la selección del educador. Se realizó y presentó una versión intermedia del programa contando únicamente con un conjunto de palabras para posteriormente implementar la versión final del programa.

\section{RESULTADOS Y DISCUSIÓN}

La aplicación que se ha desarrollado se centra en favorecer la ruta directa, es decir, el reconocimiento global de palabras. Está pensada para niños capaces de leer sílabas, pero con dificultades para leer palabras de forma global, que se manifiestan en la lentitud de lectura y en el escaso efecto de facilitación de las palabras frecuentes, es decir, leen con la misma escasa habilidad una palabra extraña que una muy conocida. El uso del programa es totalmente intuitivo y no exige ninguna necesidad ni preparación por parte del educador ni del propio usuario. En primer lugar, se deberá decidir en la configuración: 1) el conjunto de palabras para realizar el ejercicio; 2) el tiempo en milésimas de segundo que cada palabra permanecerá en la pantalla; y 3) opcionalmente si el ejercicio usa anticipador. El menú principal muestra los distintos conjuntos de palabras con los que puede trabajar el usuario final, así como la configuración de los ejercicios durante su ejecución (Figura 3).

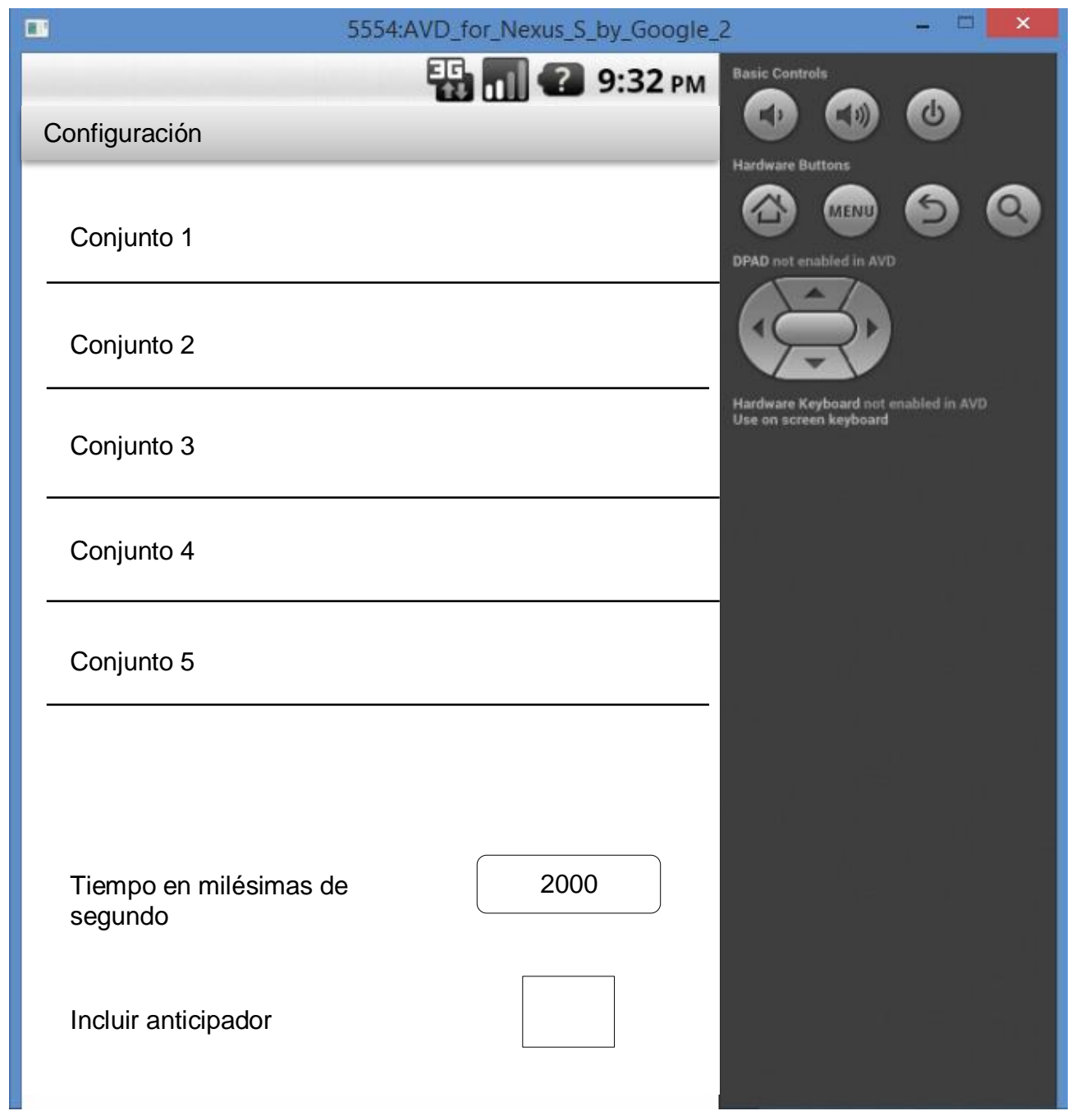

Fig. 3. Configuración del programa 
Una vez seleccionado el conjunto de palabras a trabajar, el ejercicio consiste en la identificación o lectura de diez palabras pertenecientes al conjunto seleccionado (Figura 4). Cuando el usuario del programa identifica la palabra obtiene un premio y el educador decide si continúa con otro ejercicio o acaba la tarea (Figura 5). En la parte inferior de la pantalla el programa muestra el progreso del alumno. En este desarrollo se han incluido tanto refuerzos auditivos como visuales.

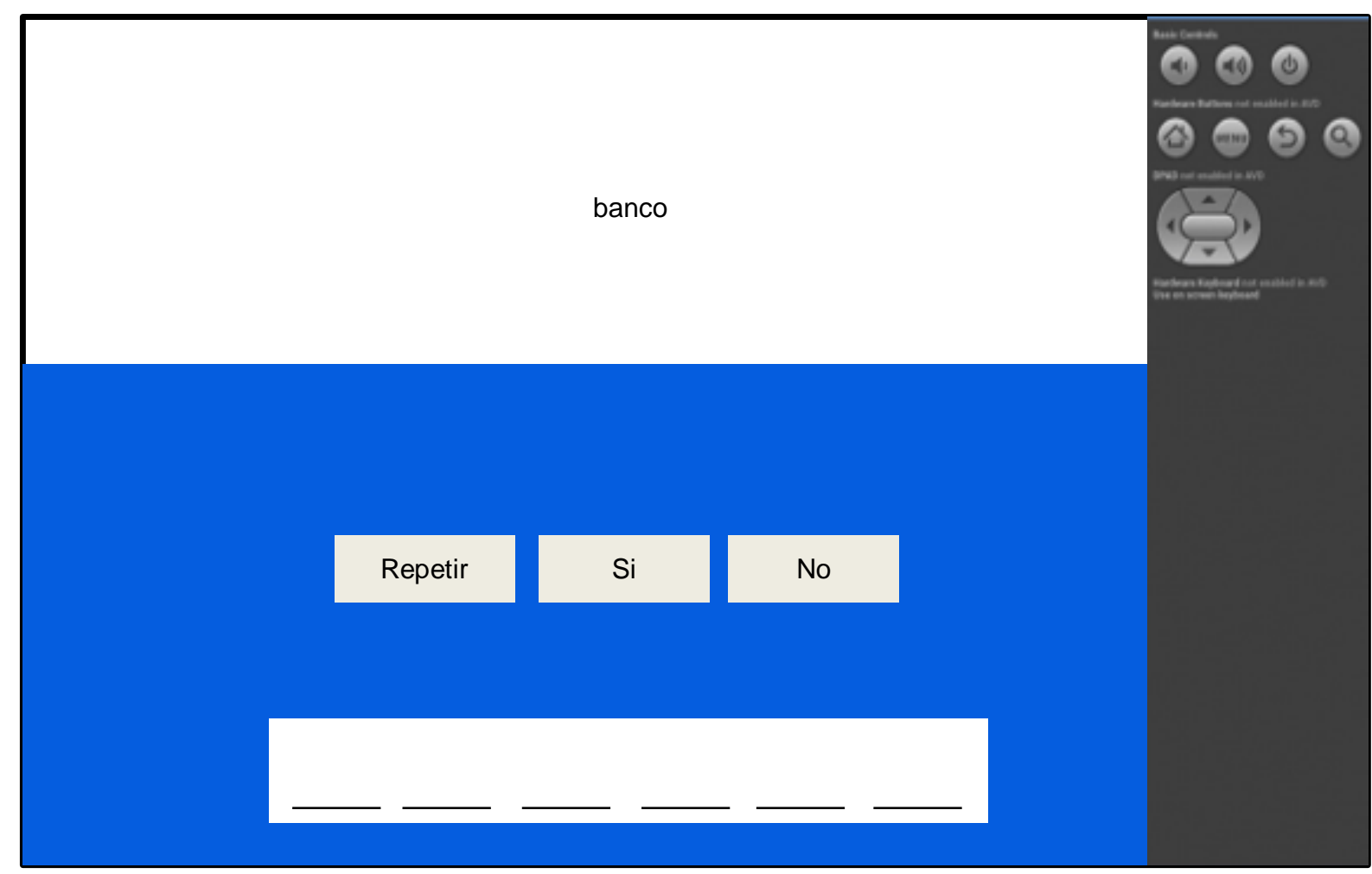

Fig. 4. Presentación de palabras

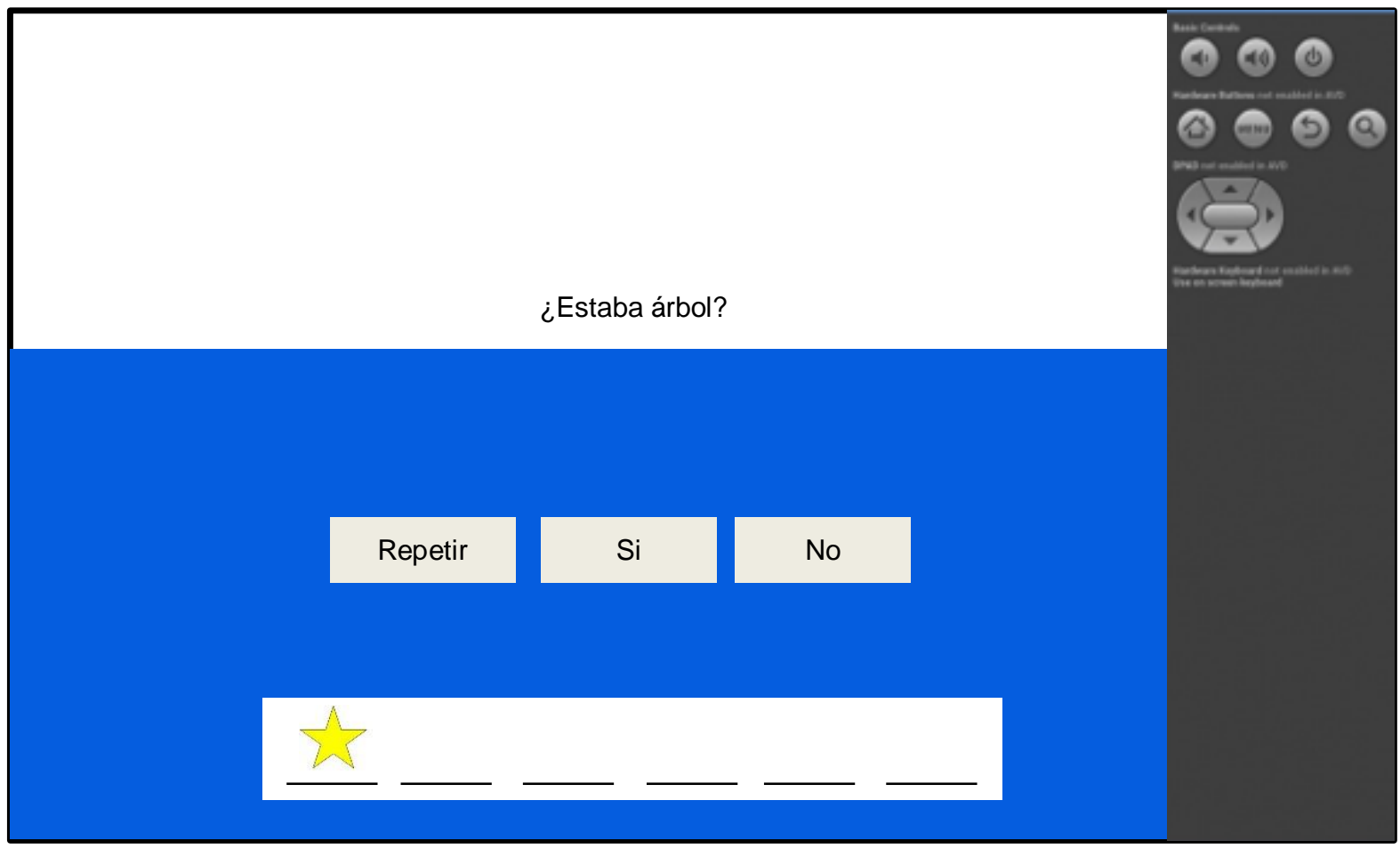

Fig. 5. Ejercicio de identificación de palabras

La aplicación que se ha desarrollado manipula dos variables para entrenar a los usuarios en el proceso de reconocimiento global de las palabras: la restricción del tiempo de lectura y la anticipación mental. A pesar de lo que podría parecer, la mirada de los lectores no transita de forma continua a lo largo del texto, sino que avanza a saltos entre fijación ocular y fijación ocular. A este fenómeno se le denomina movimientos sacádicos 
(Frey y Bosse, 2018). El cerebro extrae información de los instantes en que la vista se fija en un punto y no puede utilizar los tiempos en el que el ojo se mueve. Los lectores poco expertos obtienen menos información de cada fijación ocular y a menudo retroceden para verificar o corregir. Los expertos, por el contrario, parecen guiados por la comprensión de lo que leen y su experiencia de descodificación para elegir estratégicamente sus fijaciones que les resultan más útiles. Aunque la lectura se produce habitualmente en textos con muchas palabras, la presentación controlada de palabras aisladas puede ser un buen recurso para mejorar los procesos de reconocimiento de palabras.

La aplicación presenta la palabra en una zona de la pantalla fija, favoreciendo de esta forma el proceso de dirección de la mirada y de fijación ocular. El instructor controla los milisegundos de presentación de la palabra. Esa limitación impide que la persona realice una estrategia de descodificación de las letras desde la izquierda a la derecha y favorece el fenómeno de reconocimiento global de la palabra en la post-imagen que queda en la mente tras la desaparición súbita de la imagen. La sensación que se tiene es de saber lo que ponía, aunque no se haya tenido tiempo para leer la palabra. Lógicamente el sistema no es eficaz si el tiempo de presentación es insuficiente para la fijación ocular efectiva. El educador deberá tantear el mínimo tiempo que necesita cada usuario para reconocer lo más rápido posible la palabra por la ruta directa, impidiendo que puede se proceda a los mecanismos propios de la ruta indirecta.

El segundo recurso que ofrece nuestra aplicación se basa en el fenómeno mental de anticipación cognitiva mediante el cual se facilitan los procesos de reconocimiento sensorial si de antemano buscamos una cierta información (Foucart et al., 2015). A este fenómeno se le conoce como procesamiento arriba-abajo. En nuestro caso, los procesos de reconocimiento sensorial son la identificación de los caracteres de la palabra y lo facilitamos activando previamente la palabra y su significado. La tarea comienza con un activador: el niño escucha o lee una palabra que debe almacenar en su memoria. A continuación, se presenta una palabra escrita y se le pide que reconozca si es o no es la que previamente ha almacenado. Este mecanismo es un activador y facilitador del reconocimiento y lo combinamos con la limitación del tiempo de fijación ocular, impidiendo la ruta indirecta o subléxica. Esperamos que la manipulación de la variable de tiempo de lectura y la facilitación de la anticipación incremente la eficacia de la ruta directa en los niños que se entrenen con la aplicación.

\section{CONCLUSIONES}

Del presente trabajo se pueden obtener las siguientes conclusiones: 1) El desarrollo de software presentado puede ayudar en el proceso de reconocimiento automático de palabras en niños con dificultades de aprendizaje de la lectura. Persigue mejorar su reconocimiento automático del léxico mediante el entrenamiento sistemático de la ruta directa de lectura o ruta global; 2) La aplicación es compatible en tabletas con sistemas operativos Android y proporciona una solución de enseñanza flexible que permite un aprendizaje personalizado a las necesidades de cada alumno; y 3) La aplicación posibilita que los educadores puedan controlar con facilidad las variables determinantes en el procesamiento cognitivo de los ejercicios del entrenamiento: la duración de los estímulos, que determina la posibilidad de uso o no de la ruta indirecta o subléxica; las ayudas propias del procedimiento de anticipación cognitiva, que facilita el procesamiento de la información en el sentido arriba-abajo; y la retroalimentación al usuario final, que refuerza y motiva el aprendizaje.

\section{REFERENCIAS}

Alarcón-Aldana, A. C., Urrutia-Pinilla, J., y Callejas-Cuervo, M., Aplicación Móvil para la Administración de Variables Físicas en Ciclismo al Aire Libre, http://doi.org/10.4067/S0718-07642016000400019, Información Tecnológica, 27(4), 175-182 (2016)

Alonzo, C. N., Mcllraith, A. L., Catts, H. W., y Hogan, T. P., Predicting Dyslexia in Children With Developmental Language Disorder, https://doi.org/10.1044/2019_JSLHR-L-18-0265, Journal of Speech, Language and Hearing Research, 63(1), 151-162 (2020)

Asociación Estadounidense de Psiquiatría, Manual diagnóstico y estadístico de los trastornos mentales (DSM-5), $5^{\mathrm{a}}$ ed., Editorial Médica Panamericana S. A., Madrid, España (2014)

Cáceres, L., y Ossandón, A., Desarrollo de una aplicación móvil para reconocimiento de personas después de una catástrofe natural utilizando la tecnología NFC, http://doi.org/10.4067/S0718-33052018000500028, Ingeniare Revista Chilena de Ingeniería, 26, 28-44 (2018)

Castro-Rivera, V. P., Herrera-Acuña, R. A., y Villalobos-Abarca, M. A., Desarrollo de un software web para la generación de planes de gestión de riesgos de software, http://doi.org/10.4067/S0718-07642020000300135, Información Tecnológica, 31(3), 135-148 (2020)

Chiang, H.Y., y Liu, C.H., Evaluation of the benefits of assistive reading software: perceptions of high school students with learning disabilities, https://doi.org/10.1080/10400435.2011.614673, Assistive Technology, 23(4), 199-204 (2011) 
Choque-Tolmo, B. M., Villalobos-Abarca, M. A., y Herrera-Acuña, R. A., Desarrollo de un software web para la gestión de planes de negocios, http://doi.org/10.4067/S0718-07642020000400045 Información Tecnológica, 31(4), 45-60 (2020)

Dawson, K., Antonenko, P., Lane, H., y Zhu, J., Assistive Technologies to Support Students with Dyslexia, https://doi.org/10.1177/0040059918794027, Teaching Exceptional Children, 51(3), 226-239 (2019)

Foucart, A., Ruiz-Tada, E., y Costa, A., How do you know I was about to say "book"? Anticipation processes affect speech processing and lexical recognition, https://doi.org/10.1080/23273798.2015.1016047,Language, Cognition and Neuroscience, 30(6), 768-780 (2015)

Frey, A., y Bosse, M., Perceptual span, visual span, and visual attention span: Three potential ways to quantify limits on visual processing during reading, https://doi.org/10.1080/13506285.2018.1472163, Visual Cognition, 26(6), 412-429 (2018)

Fuentes, J. L., Albertos, J., y Torrano, F., Hacia el Mobile-Learning en la escuela: Análisis de factores críticos en el uso de las tablets en centros educativos españoles, https://doi.org/10.14201/eks2019_20_a3, Education in the Knowledge Society (EKS), 20, 17-17 (2019)

Galuschka, K., Görgen, R., y otros 3 autores, Effectiveness of spelling interventions for learners with dyslexia: A metaanalysis and systematic review, https://doi.org/10.1080/00461520.2019.1659794, Educational Psychologist, 55(1), 1-20 (2020)

Gou, H., y Dezuanni, M., Hacia la comprensión de las vidas digitales de los niños en China y Australia, https://doi.org/10.3916/C57-2018-08, Comunicar: Revista Científica de Comunicación y Educación, 26(57), 81-90 (2018)

Máñez-Carvajal, C., Evaluación de accesibilidad web de las universidades chilenas, https://doi.org/10.4067/S071850062020000500069, Formación Universitaria, 13(5), 69-76 (2020)

Máñez-Carvajal, C., y Máñez, C., Adquisición de vocabulario básico I, Aquari, 2(1), 1-3 (2020)

Martínez-García, C., Suárez-Coalla, P., y Cuetos, F., Development of orthographic representations in Spanish children with dyslexia: The influence of previous semantic and phonological knowledge, https://doi.org/10.1007/s11881-01900178-6, Annals of Dyslexia, 69(2), 186-203 (2019)

Morales, J. C., Ramírez, N. E., Vargas, S. H., y Peñuela, A. J., Uso de aplicativos móviles en el aula y sus factores determinantes, http://doi.org/10.4067/S0718-50062020000600013, Formación Universitaria, 13(6), 13-22 (2020)

Orellana, A. M., Oyarzún-Díaz, P. A., Briones-Rojas, C., y Vidal-Silva, C. L., Prototipo de simulador de audiometría para estudiantes de fonoaudiología de la Universidad Santo Tomás, Chile, http://doi.org/10.4067/S0718-

50062020000200003, Formación Universitaria, 13(2), 3-10 (2020)

Peters, J. L., De Losa, L., Bavin, E. L., y Crewther, S. G., Efficacy of dynamic visuo-attentional interventions for reading in dyslexic and neurotypical children: A systematic review, https://doi.org/10.1016/j.neubiorev.2019.02.015, Neuroscience \& Biobehavioral Reviews, 100, 58-76 (2019)

Pressman, R., Ingeniería del software: un enfoque práctico, $7^{a}$ ed., Mc-Graw Hill interamericana, México D.F., México (2010)

Skiada, R., Soroniati, E., Gardeli, A., y Zissis, D., EasyLexia: A Mobile Application for Children with Learning Difficulties, https://doi.org/10.1016/j.procs.2014.02.025, Procedia Computer Science, 27, 218-228 (2014)

Snowling, M. J., y Hulme, C., The science of reading: A handbook, $1^{\underline{a}}$ ed., Blackwell Publishing, Victoria, Australia (2005) Sommerville, I., Ingeniería del software, $7^{a}$ ed., Pearson Educación S. A., Madrid, España (2005)

Tariq, R., y Latif, S., A Mobile Application to Improve Learning Performance of Dyslexic Children with Writing Difficulties, Journal of Educational Technology and Society, 19(4), 151-166 (2016)

Tressoldi, P. E., Vio, C., y lozzino, R., Efficacy of an Intervention to Improve Fluency in Children with Developmental Dyslexia in a Regular Orthography, https://doi.org/10.1177/00222194070400030201, Journal of Learning Disabilities, 40(3), 203-209 (2007)

Tucci, R., Savoia, V., Bertolo, L., Vio, C., y Tressoldi, P. E. Efficacy and efficiency outcomes of a training to ameliorate developmental dyslexia using the online software Reading Trainer, BPA Applied Psychology Bulletin, 273(64), 53-60 (2015)

Kast, M., Baschera, G.M., y otros tres autores, Computer-Based Learning of Spelling Skills in Children with and without Dyslexia. Annals of Dyslexia, 61(2), 177-200 (2011)

West, M., y Vosloo, S. E., Directrices de la UNESCO para las políticas de aprendizaje móvil, $1^{\text {a }}$ ed., Organización de las Naciones Unidas para la Educación, la Ciencia y la Cultura, París, Francia (2013) 